Document downloaded from:

http://hdl.handle.net/10251/36765

This paper must be cited as:

Cerdá Alabern, L.; Darehshoorzadeh, A.; Pla, V. (2013). Optimum Node Placement in Wireless Opportunistic Routing Networks. Ad Hoc Networks. 11(8):2273-2287. doi:10.1016/j.adhoc.2013.05.010.

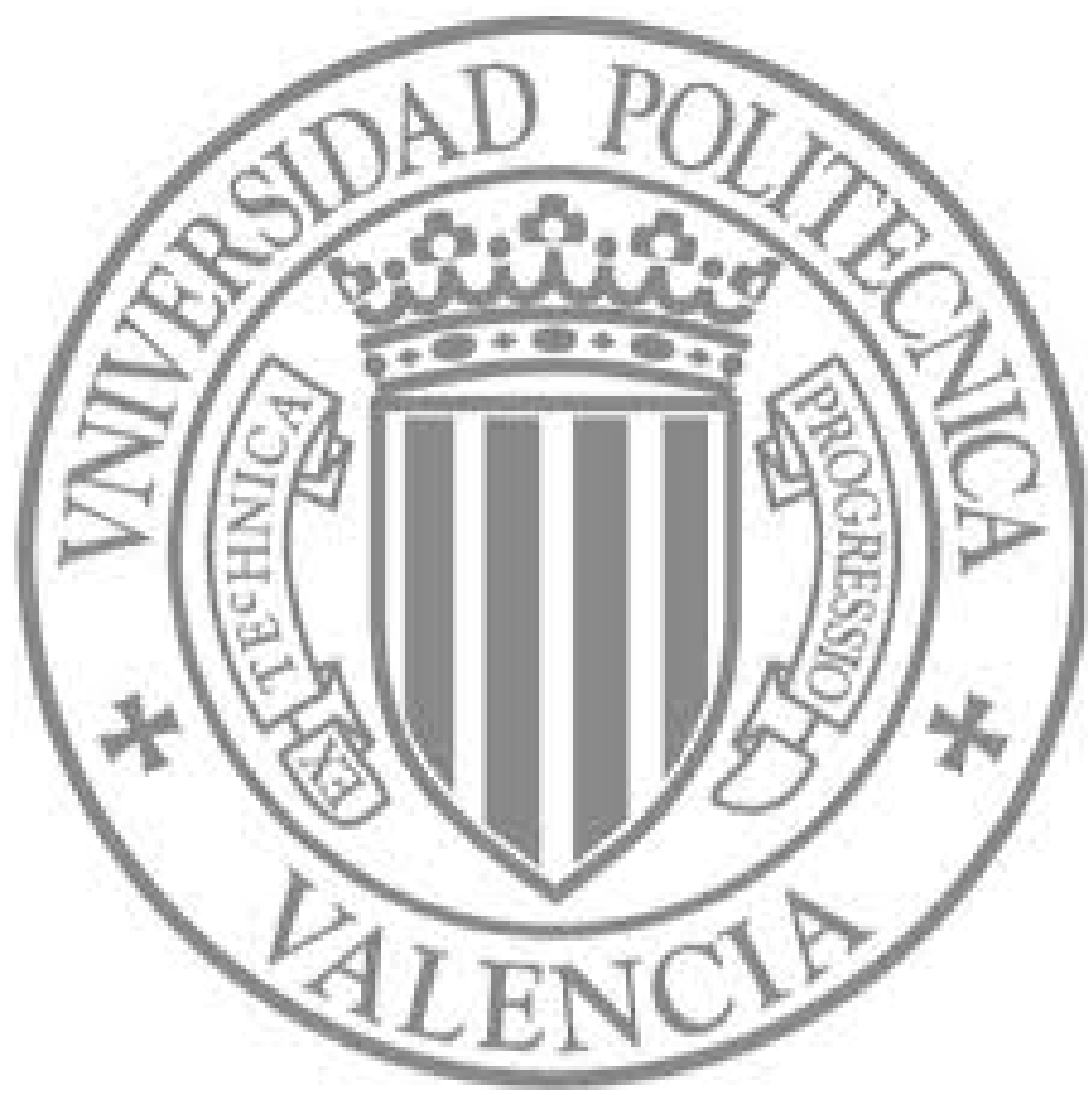

The final publication is available at

http://dx.doi.org/10.1016/j.adhoc.2013.05.010

Copyright Elsevier 


\title{
Optimum Node Placement in Wireless Opportunistic Routing Networks
}

\author{
Llorenç Cerdà-Alabern, Amir Darehshoorzadeh ${ }^{\mathrm{a}}$, Vicent Pla ${ }^{\mathrm{b}}$ \\ ${ }^{a}$ Univ. Politècnica de Catalunya \\ Computer Architecture Dep. \\ Barcelona, Spain \\ Email: \{llorenc,amir\}@ac.upc.edu \\ ${ }^{b}$ Univ. Politècnica de València \\ Communications Dep. \\ València, Spain \\ Email:vpla@dcom.upv.es
}

\begin{abstract}
In recent years there has been a growing interest in Opportunistic Routing as a way to increase the capacity of wireless networks by exploiting its broadcast nature. In contrast to traditional uni-path routing, in opportunistic routing the nodes overhearing neighbor's transmissions can become candidates to forward the packets towards the destination.

In this paper we address the question: What is the maximum performance that can be obtained using opportunistic routing? To answer this question we use an analytical model that allows to compute the optimal position of the nodes, such that the progress towards the destination is maximized. We use this model to compute bounds to the minimum expected number of transmissions that can be achieved in a network using opportunistic routing.
\end{abstract}

Keywords: wireless networks; opportunistic routing; maximum performance; analytical model.

\section{Introduction}

Multi-hop wireless networks (MWN) [1, 2] have become a very active research field during the last years. Routing in MWN is more challenging than in wired networks because of two fundamental differences. The first difference is the heterogeneous characteristics of wireless links. As a consequence, there can be significant differences in packet delivery probabilities across the links of a MWN network. The second difference is the broadcast nature of wireless transmissions [3]. Unlike wired networks, where links are typically point to point, when a node transmits a packet in a wireless network the neighbors of the the intended destination node can overhear it.

Routing protocols in MWN have traditionally managed the heterogeneous characteristics of wireless links by using distributed protocols that at each node choose the best link for every destination (referred to as next-hop). Once all next-hops have been chosen, all packets between a source and a destination follow the same path. This motivates the name of uni-path routing for such type of protocols.

Opportunistic Routing (OR) $[4,5,6,7]$ has been proposed to increase the performance of MWNs by taking advantage of the broadcast nature of the wireless medium. In OR, instead of preselecting a single node to be the next-hop as a forwarder for a packet, 
an ordered set of nodes (referred to as candidates) is selected as the potential next-hop forwarders. Thus, the source can use multiple potential paths to deliver the packets to the destination. After the transmission of a packet, all the candidates that successfully receive it will coordinate among themselves to determine which one will actually forward it; the others will simply discard the packet.

Previous research in this field has principally concentrated on proposing and evaluating different candidate selection mechanisms and routing protocols. Then the performance of the proposed mechanism is compared with that of the baseline scenario of traditional unipath routing or with that of other OR mechanisms. Performance is generally measured in terms of the expected number of transmissions from the source to the destination (which, like in [8], we refer to as expected any-path transmission, EAX). To the best of our knowledge, all studies assume that the network topology is given and the evaluations and comparisons are done over that topology, or a variety of them. In this paper we follow a different approach. Here, we investigate the maximum gain that can be obtained by using OR. For this purpose, we study the optimal position of the nodes acting as candidates. The obtained insight is then applied to propose practical design rules for multihop wireless networks.

In the first part of the paper, we address the question: What is the maximum gain that can be obtained using OR? We shall refer to gain as the relative difference of the expected number of transmissions required between OR and the baseline uni-path routing scenario. More specifically, we focus on a scenario where the maximum number of candidates per node is limited. To answer the former question we use a network where the nodes are optimally located so that at each transmission the progress towards the destination is maximized. To do so, we shall assume that we have a formula for the delivery probability, $p(d)$, between nodes at a distance $d$. For the sake of simplicity we shall assume the same function $p(d)$ for any pair of nodes. The model, however, could be generalized assuming a different function for every link. In our analysis $p(d)$ will be given by the radio propagation model. An expression to compute the expected number of transmissions in OR has been obtained by several authors (e.g., $[8,9,10])$. That expression is recursive and has a non-linear dependence on the delivery probability between the nodes. We shall use this formula for comparison purposes, and in the rest of the paper we shall refer to it as the EAX recursive formula.

Due to the complex form of the EAX recursive formula, even if $p(d)$ is known, it may not give a feasible way to derive the optimal position, and thus, the maximum OR gain. We solve this problem by computing the optimal position of the nodes maximizing the progress towards the destination when OR is used. The same principle was in used in [11] in the context of uni-path routing. We show that this approach allows deriving a set of equations that can be solved numerically in order to compute the optimal distances between a node and its candidates. We refer to these distances as the maximum progress distances (MPD). Additionally, by maximizing the progress towards the destination we establish a tight lower bound on the expected number of transmissions in OR, and thus, an upper bound on the OR gain.

Studying the optimal position of the candidates is theoretically insightful, and also allow us to assess the maximum performance achievable with OR and to establish lower and upper bounds. Moreover, from a practical perspective there are also a number of implications that are derived from the results in our study with application, for instance, to network deployment and design of routing protocols.

Obviously, to apply our results to deploy future wireless multi-hop network it is nec- 
essary to be able to decide, to some extent, where the nodes are to be placed. However, it is not necessary a complete freedom to place nodes (i.e., it is not necessary to place the node at the very precise positions given by the MPDs) since our results reveal a rather low sensitivity of the performance with respect to deviation from the optimal positions. Therefore, although not optimal, a network design based on the MDPs and the existing node positioning constraints can still provide a good design.

Furthermore, in a practical wireless multi-hop network design, the total number of nodes to be deployed is also a very important practical issue. Even if a limited number of candidates per node is considered, building a network in which each node has neighbors located precisely at the MPD would imply, in general, a network with a very large or infinite number of nodes. By applying the insight gained from the analysis of the MPDs, we propose practical design rules based on regular one- and two-dimensional topologies. The proposed solutions exhibit a performance that is very close to the optimal one, whereas the number of nodes is greatly reduced.

Another important area of potential application of the results in this paper is that of routing protocols for wireless multi-hop networks. The MPDs can be used for routing protocols in OR that are based in the geographic routing principle. In geographic routing, the position of the nodes and that of the destination is used in the routing decisions. In wireless multi-hop networks, it is considered that routing protocols that are not based on geographic routing are not scalable [12]. Uni-path routing schemes based on geographic routing often use a greedy approach by forwarding data packets to the neighbor geographically closest to the destination [13]. This principle has also been applied in OR [14], where the distance from the candidates that have received that packet and the destination is used to decide which candidate will forward the packet onto the next hop. However, a basic premise in this paper is that, in order to keep within practical limits the overhead due to candidate coordination, the number of candidates per node must be limited and rather small. The MPDs can be used to select the appropriate candidate for each node.

Finally, from a practical perspective it also worth mentioning that our results show that instead of needing the values of the MDPs, which depend on the propagation characteristics, and the distances to the neighboring nodes, a node can select its candidate set by simply estimating the delivery probabilities to its neighbors.

The remainder of this paper is organized as follows. In Section 2 we study the positions of the candidates that yield the maximum progress per transmission, and the results are used in Section 3 to derive bounds for the expected number of transmission necessary to reach the destination. Section 4 introduces the propagation model that we have used in the numerical experiments presented in Section 5. In Section 6 we describe a procedure to construct a network using the maximum progress distances. The expected number of transmissions in this network yields a tight upper bound of the optimal value. Section 7 compares the MPD with the optimal distances. In Section 8 a practical method is proposed to achieve a performance close the optimum one with a much lower number of nodes. Section 9 investigates this method when applied to a two-dimensional grid. Finally, Section 10 surveys the related work and concluding remarks are given in Section 11.

\section{Optimal Positions of Candidates}

We study the position of the candidates in order to maximize the progress towards the destination. The ingredients of our model are: the maximum number of candidates per node $n$; and the formula for the delivery probability at a distance $d, p(d)$, which we suppose to be the same for all the nodes. Assume that the destination is far from a generic 


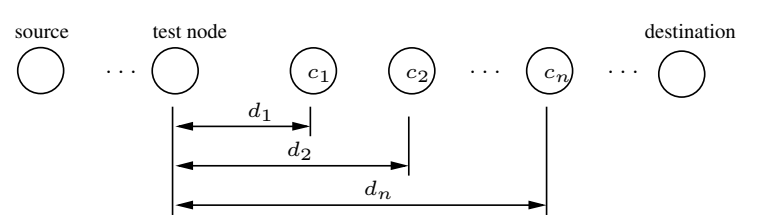

Figure 1: Test node and its candidates.

test node for whose candidates are looking for. Clearly, the optimum candidates will be located over the segment between the test node and the destination (see Figure 1).

Let $\left\{c_{1}, c_{2}, \cdots c_{n}\right\}$ be the ordered set of candidates of the generic test node $\left(c_{n}\right.$ has the highest priority, and $c_{1}$ the least one), and let $d_{i}$ be the distance from the test node to the candidate $c_{i}$ (see Figure 1). We assume that a coordination protocol exist among the candidates, such that the highest priority candidate receiving the packet will forward the packet (if it is not the destination), while the other nodes will simply discard it. Assume that $p\left(d_{i}\right)$ is the delivery probability from the test node to the candidate $c_{i}$, and let $\Delta_{n}$ be the random variable equal to the distance reached after one transmission shot. Clearly,

$$
\mathrm{E}\left[\Delta_{n}\right]=d_{n} p\left(d_{n}\right)+d_{n-1} p\left(d_{n-1}\right)\left(1-p\left(d_{n}\right)\right)+\cdots+d_{1} p\left(d_{1}\right) \prod_{i=2}^{n}\left(1-p\left(d_{i}\right)\right)
$$

That is, the packet will progress a distance $d_{n}$ if the highest priority candidate $c_{n}$ receives it, or a distance $d_{i}, i=1, \cdots n-1$ if $c_{i}$ receives it, and no higher priority candidates receive the packet. A key observation is that taking the common factor $\left(1-p\left(d_{n}\right)\right)$ in Equation (1) we get the recursive equation:

$$
\mathrm{E}\left[\Delta_{n}\right]=d_{n} p\left(d_{n}\right)+\left(1-p\left(d_{n}\right)\right) \mathrm{E}\left[\Delta_{n-1}\right]=\mathrm{E}\left[\Delta_{n-1}\right]+\left(d_{n}-\mathrm{E}\left[\Delta_{n-1}\right]\right) p\left(d_{n}\right) .
$$

We are interested in looking for the value $d_{n}$ that maximizes Equation (2). Note that this value also maximizes the function

$$
f(x)=(x-a) p(x)
$$

where $a=\mathrm{E}\left[\Delta_{n-1}\right]$. Notice that $f(a)=0$ and $f(x)$ is increasing in the neighborhood of $a$. We shall assume that the delivery probability $p(x)$ is differentiable and $\lim _{x \rightarrow \infty} x p(x)=0$. Note that this last condition is necessary for the expected value of the distance reachable in a transmission shot to be finite. Since the delivery probability is $p(x) \geq 0$, and must be monotonically decreasing (the higher is the distance, the lower the delivery probability), we conclude that (3) must have a point $x^{*} \in(a, \infty)$ for which $f$ is increasing for $x \in$ $\left(a, x^{*}\right)$, and decreasing for $x \in\left(x^{*}, \infty\right)$. In order words, the function $f(x)$ is quasiconcave in $x \in(a, \infty)$, having a unique critical point equal to its global maximum in this interval. Additionally, since $d_{i-1}<d_{i}(i=2, \cdots, n)$, it is $\mathrm{E}\left[\Delta_{n-1}\right]<d_{n-1}<d_{n}$. Thus, we can reduce the optimization domain to $d_{n} \in\left(d_{n-1}, \infty\right)$. Under these conditions we can compute the distances $d_{i}(i=1, \cdots, n)$ that maximize (2) by solving:

$$
\frac{\partial \mathrm{E}\left[\Delta_{i}\right]}{\partial d_{i}}=0, \quad d_{i} \in\left(d_{i-1}, \infty\right), i=1, \cdots, n
$$

which gives the set of equations:

$$
p\left(d_{i}\right)+\left(d_{i}-\mathrm{E}\left[\Delta_{i-1}\right]\right) p^{\prime}\left(d_{i}\right)=0, d_{i} \in\left(d_{i-1}, \infty\right), i=1, \cdots, n
$$


where $\mathrm{E}\left[\Delta_{0}\right]=0$ and $d_{0}=0$. Note that by using Equation (4) we can compute $d_{1}$ from $p\left(d_{1}\right)+p^{\prime}\left(d_{1}\right) d_{1}=0$. Then, substituting in (2) we have $\mathrm{E}\left[\Delta_{1}\right]=d_{1} p\left(d_{1}\right)$, which can be used to compute $d_{2}$ using (4) and so on until $d_{n}$. We shall refer to these distances as the MPD. In the sequel we shall refer to them as $d_{1}, \cdots, d_{n}$, and denote the expected number of transmissions given by Equation (2) using these distances as $\mathrm{E}\left[\Delta_{n}^{*}\right]$. Note also that a consequence of Equation (4) is that the MPD for the already existing candidates do not change if we decide to add a new candidate to the candidate set.

Note that the previous model, could be generalized by assuming a different delivery probability for every candidate of the source $s: p_{s i}(x)$. Of course, in this case the MPD to the candidates could be different for every node.

\section{Maximum Performance of OR}

In this section we investigate the performance of OR in terms of the expected number of transmissions to send a packet from the source to the destination, i.e. in terms of the EAX. To do so, we define $\tau_{n}$ to be the random variable equal to the number of transmissions required to send a packet from the source to the destination using at most $n$ candidates per node. We shall use the notation $E A X_{n}=\mathrm{E}\left[\tau_{n}\right]$ to refer to the value of EAX in a network with this constraint. Note that $n=1$ correspond to traditional uni-path routing. We are thus interested in obtaining bounds to $E A X_{n}$.

\subsection{Infinite Number of Candidates}

We first derive a result that will be useful in the bounds derived afterwards. Assume an infinitely dense network where the nodes can choose an infinite number of candidates. Assume further that there is not limitation on the minimum delivery probability that living links can have. Let $\tau_{\infty}$ be the random variable equal to the number of transmissions required to send a packet from the source to the destination in such network. With these assumptions, some node as close to the destination as we want can receive the packet with probability 1 (we can choose a region arbitrarily close to the destination that contains an infinite number of candidates). Therefore, if the destination does not receive the packet after it is firstly transmitted by the source, by the previous reasoning, one candidate arbitrarily close to it will have received it, and will relay it to the destination with just one more transmission. Thus, in this case, $\tau_{\infty}=2$. Let $D$ be the distance between the source and the destination. From the previous discussion we conclude that:

$$
\mathrm{E}\left[\tau_{\infty}\right]=p(D)+2(1-p(D))=2-p(D)
$$

\subsection{A Lower Bound for the Expected Number of Transmissions}

Assume a network with $n$ candidates per node. Since $\mathrm{E}\left[\Delta_{n}^{*}\right]$ computed in Section 2 using the MPD given by equations (4) is the maximum progress towards the destination after every transmission shot, we have that the expected number of transmissions to send a packet from the source to the destination $\left(\mathrm{E}\left[\tau_{n}\right]\right)$ is lower bounded as follows

$$
\mathrm{E}\left[\tau_{n}\right] \geq \frac{D}{\mathrm{E}\left[\Delta_{n}^{*}\right]}
$$

where $D$ is the distance between the source and the destination.

The bound given by Equation (6) will be tight as long as the distance $D$ is large compared with $d_{n}$, and the nodes are located at the MPD. Clearly, for those nodes that 
are closer than $d_{n}$ to the destination, the optimal positions cannot be given by the MPD. In this case the highest priority candidate will be the destination. Thus, the distance of the highest priority candidate will be the distance to the destination, and the optimal position of the other candidates should be computed taking the distances that minimize the EAX. In fact, this "boundary effect" will propagate to the position of the other nodes between the source and the destination, and their optimal positions may be slightly different than those obtained using the MPD (we shall investigate this in Section 7). Nevertheless, the expected distance progress after each transmission could not be as high as the one obtained using the MPD, which guarantees that (6) is a lower bound.

We can use the result obtained for an infinite number of candidates to improve the bound given by (6). First, the expected number of transmissions cannot be less that the value given by Equation (5). Therefore, we have that:

$$
\mathrm{E}\left[\tau_{n}\right] \geq \max \left(2-p(D), \frac{D}{\mathrm{E}\left[\Delta_{n}^{*}\right]}\right)
$$

The bound given by (7) can still be improved as we explain next. As we said before, when the nodes are closer than $d_{n}$ to the destination, the position of the nodes cannot be the MPD. Therefore, using $\mathrm{E}\left[\Delta_{n}^{*}\right]$ as the progress in this region may be a coarse approximation. To estimate the progress in this region we note that before the packet reaches the destination, at least one node in the interval $\left[D-d_{n}, D\right)$ will receive it, because the furthest candidate of any node is at a distance $d_{n}$. We shall refer to the first node in this interval that receives the packet as $v(x)$, where $x$ is the distance from this node to the destination. Now, the number of transmissions from the source to $v(x)$ can be lower bounded by $(D-x) / \mathrm{E}\left[\Delta_{n}^{*}\right]$ (i.e. assuming the maximum progress), and the number of transmissions from $v(x)$ to the destination can be lower bounded assuming an infinite number of candidates between $v(x)$ and the destination (Equation (5)). Adding both terms we have $\mathrm{E}\left[\tau_{n} \mid v(x)\right] \geq(D-x) / \mathrm{E}\left[\Delta_{n}^{*}\right]+2-p(x)=D / \mathrm{E}\left[\Delta_{n}^{*}\right]+2-p(x)-x / \mathrm{E}\left[\Delta_{n}^{*}\right]$. Thus, if we want a lower bound we must take $x$ that minimizes $\mathrm{E}\left[\tau_{n} \mid v(x)\right]$ in the interval $x \in\left(0, d_{n}\right]$.

Summing up, we have that:

$$
E A X_{n}=\mathrm{E}\left[\tau_{n}\right] \geq \max \left(2-p(D), \frac{D}{\mathrm{E}\left[\Delta_{n}^{*}\right]}+\inf _{x \in\left(0, d_{n}\right]}\left\{2-p(x)-\frac{x}{\mathrm{E}\left[\Delta_{n}^{*}\right]}\right\}\right)
$$

\subsection{An Upper Bound for the Gain}

Let us denote by $\tau_{n}^{*}$ the number of transmissions when the candidates are optimally placed. In order to measure the improvement that can be reached using OR we define the gain $\left(G_{n}\right)$ as the relative difference of the expected number of transmissions required with the OR with $n$ candidates $\left(\mathrm{E}\left[\tau_{n}^{*}\right]\right)$, with respect to the uni-path routing case. Note that OR with only 1 candidate per node is equivalent to uni-path routing. Therefore, we shall refer to the expected number of transmissions with uni-path routing as $\mathrm{E}\left[\tau_{1}^{*}\right]$, and thus:

$$
G_{n}=\frac{\mathrm{E}\left[\tau_{1}^{*}\right]-\mathrm{E}\left[\tau_{n}^{*}\right]}{\mathrm{E}\left[\tau_{1}^{*}\right]}=1-\frac{\mathrm{E}\left[\tau_{n}^{*}\right]}{\mathrm{E}\left[\tau_{1}^{*}\right]}
$$

Using the same intuition as in (6) we can write

$$
\frac{D}{\mathrm{E}\left[\Delta_{1}^{*}\right]} \leq \mathrm{E}\left[\tau_{1}^{*}\right] \leq \frac{\left\lceil D / d_{1}\right\rceil d_{1}}{\mathrm{E}\left[\Delta_{1}^{*}\right]}
$$


and using the lower bound for $\mathrm{E}\left[\tau_{n}^{*}\right]$ and the upper bound for $\mathrm{E}\left[\tau_{1}^{*}\right]$ it follows from (9) that

$$
G_{n} \leq 1-\frac{D / d_{1}}{\left\lceil D / d_{1}\right\rceil} \frac{\mathrm{E}\left[\Delta_{1}^{*}\right]}{\mathrm{E}\left[\Delta_{n}^{*}\right]}
$$

\section{Propagation Model}

In order to model the delivery probabilities we will assume that the channel impairments are characterized by a shadowing propagation model. This model includes deterministic path loss and large scale fading. It is a standard propagation model when considering the network capacity [15]. More specifically, the power received at a distance $d\left(P_{r}(d)\right)$, in terms of the transmitted power $\left(P_{t}\right)$ is given by:

$$
\left.P_{r}(d)\right|_{d B}=10 \log _{10}\left(\frac{P_{t} G_{t} G_{r} \lambda^{2}}{L(4 \pi)^{2} d^{\beta}}\right)+X_{d B}
$$

where $G_{t}$ and $G_{r}$ are the transmission and reception antenna gains respectively, $L$ is a system loss, $\lambda$ is the signal wavelength $\left(c / f\right.$, with $\left.c=3 \times 10^{8} \mathrm{~m} / \mathrm{s}\right), \beta$ is a path loss exponent, and $X_{d B}$ is a Gaussian random variable with zero mean and standard deviation $\sigma_{d B}$.

Packets are correctly delivered if the received power is greater than or equal to RXThresh. Note that we shall not consider collisions in our model. Thus, the delivery probability at a distance $d(p(d))$ is given by:

$$
\begin{aligned}
& p(d)=\operatorname{Prob}\left(\left.P_{r}(d)\right|_{d B} \geq 10 \log _{10}(\text { RXThresh })\right)= \\
& Q\left(\frac{1}{\sigma_{d B}} 10 \log _{10}\left(\frac{\text { RXThresh } L(4 \pi)^{2} d^{\beta}}{P_{t} G_{t} G_{r} \lambda^{2}}\right)\right)
\end{aligned}
$$

where $Q(z)=\frac{1}{\sqrt{2 \pi}} \int_{z}^{\infty} \mathrm{e}^{-y^{2} / 2} d y$.

In our numerical experiments we have set the model parameters to the default values used by the network simulator (ns-2) [16], given in Table 1. Table 2 shows typical values for $\beta$ and $\sigma_{d B}$.

Table 1: Default ns values for the shadow-

\begin{tabular}{|c|c|c|c|}
\hline \multicolumn{2}{|c|}{ Environment } & $\beta$ & $\sigma_{d B}$ \\
\hline Outdoor & $\begin{array}{l}\text { Free space } \\
\text { urban }\end{array}$ & $\begin{array}{l}2 \\
2.7 \sim 5\end{array}$ & $4 \sim 12$ \\
\hline Office & $\begin{array}{l}\text { Line-of-sight } \\
\text { Obstructed }\end{array}$ & $\begin{array}{l}1.6 \sim 1.8 \\
4 \sim 6\end{array}$ & $7 \sim 9.6$ \\
\hline
\end{tabular}
ing propagation model.

Table 2: Typical values for $\beta$ and $\sigma_{d B}$.

\begin{tabular}{lll}
\cline { 1 - 1 } Parameter & & Value \\
\cline { 1 - 1 }$P_{t}$ & & 0.28183815 Watt \\
RXThresh & & $3.652 \times 10^{-10}$ Watt \\
$G_{t}, G_{r}, L$ & & 1 \\
$f$ & $914 \mathrm{MHz}$ \\
\hline
\end{tabular}

Figure 2 depicts the delivery probability at a varying distance, for three values of the path loss exponent $(\beta)$ and a standard deviation $\sigma_{d B}=6 \mathrm{dBs}$. We shall use these values in the numerical results presented in later sections.

\section{Numerical Results}

We now give some numerical examples of the formulas derived in the previous sections. We shall assume that the delivery probability $p(d)$ is given by Equation (13). Substituting 


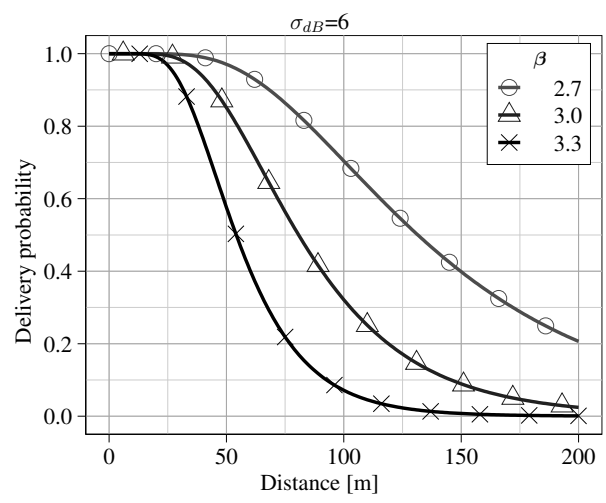

Figure 2: Delivery probability versus distance for a standard deviation $\sigma_{d B}=6 \mathrm{dBs}$.

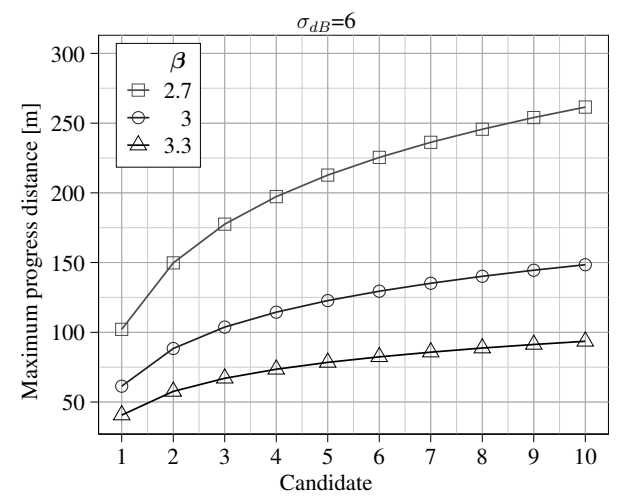

Figure 3: MPD for the candidates.

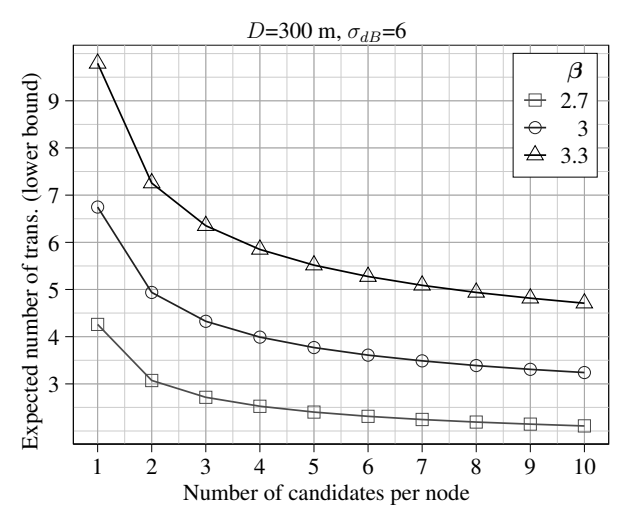

Figure 5: Expected number of transmissions, EAX (lower bound).

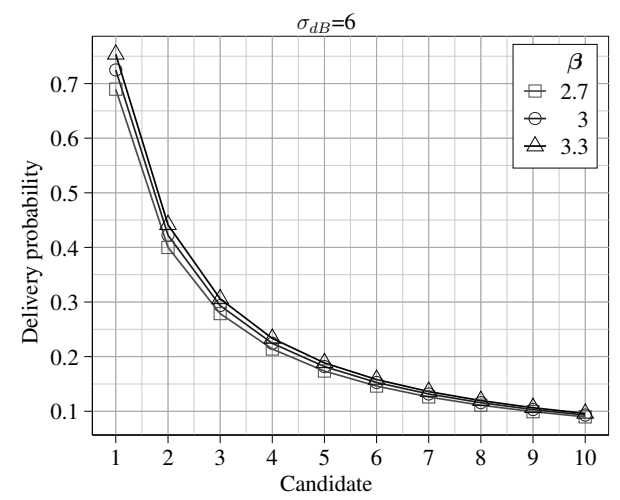

Figure 4: Delivery probability to each candidate located at the maximum progress distances.

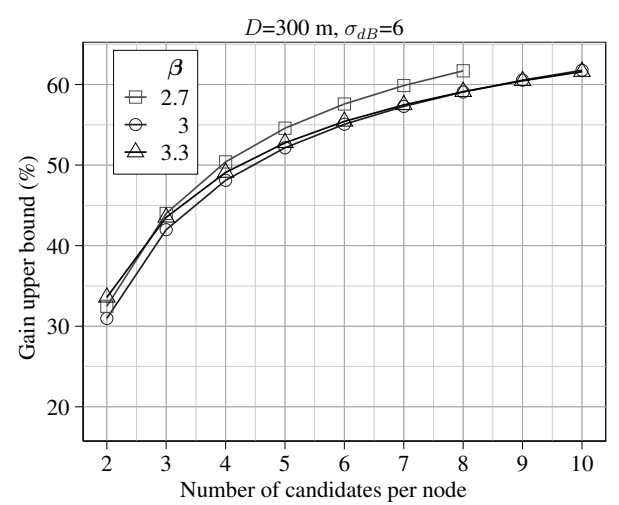

Figure 6: Gain (upper bound).

$p(d)$ in the equations (4) and solving them numerically we obtain the maximum progress distances for the candidates shown in Figure 3. The three curves correspond to three values of the loss exponent of the propagation model: $\beta=2.7, \beta=3$ and $\beta=3.3$. Note that the larger the value of $\beta$, the lower the transmission range of the nodes is, and thus, the shorter the distances to the candidates are.

Figure 4 shows the delivery probabilities obtained for the corresponding distances shown in Figure 3. It is interesting that the probabilities are very similar for all values of $\beta$. This fact could be used as a rule of thumb in the selection of candidates, or for placing the nodes in the back-haul of a mesh network.

Finally, Figure 5 depicts the lower bound of the expected number of transmissions (Equation (8)) for a distance $D=300 \mathrm{~m}$ between the source and the destination, and 


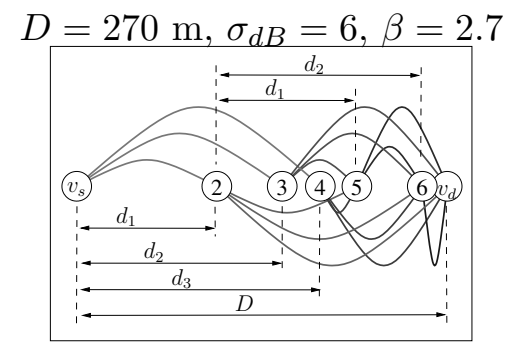

Figure 7: Quasi Optimal OR network with a maximum of 3 candidates per node.

Figure 6 shows the corresponding upper bound to the gain (Equation (11)). As we shall see in Section 6, the lower bound given by Equation (8) is very tight. Consequently, the gains that can be obtained using OR are close to the upper bound depicted in Figure 6 . These figures show that the highest gain increase occurs when we move from 1 to 2 candidates (approximately 30\% of gain). After that, the gain increases up to approximately $60 \%$ with 10 candidates. However, implementing an OR protocol with a high number of candidates is difficult, and possibly will introduce large signaling overhead and duplicated transmissions that would prevent to reach such large gains. This motivates that selecting a maximum number of candidates per node equal to 2 , or maybe 3 , is possibly a sensible choice.

\section{Quasi Optimal OR Network}

In this section we compute an upper bound for the expected number of transmissions, EAX. This bound is obtained by building a network where the candidates are positioned whenever possible using the MPD computed as in Section 2. Once the network is built, its exact EAX can be computed by using the EAX recursive formula (see e.g. [10]). Note that not all the candidates can be located using the MPD distances, since for some nodes the distance to the destination can be shorter than the distance to the candidate. For these nodes we will use as candidates the destination and its closest neighbors located between the node and the destination. Since these candidates, at least, are not located at the optimum positions, the expected number of transmissions computed for such network will be an upper bound to the minimum expected number of transmissions that can be achieved using OR. We shall refer to such network as Quasi Optimal OR (QOO) network.

Figure 7 depicts an example of a network with 3 candidates per node build using these rules. The source is $v_{s}$ and the destination is $v_{d}$. Nodes 2,3 and 4 are located at the MPD from $v_{s}: d_{1}, d_{2}$ and $d_{3}$ respectively. Nodes 5 and 6 are located at the MPD from node 2: $d_{1}$ and $d_{2}$ respectively. Since $v_{d}$ is closer from node 2 than $d_{3}, v_{d}$ is taken as the third candidate of node 2 . Since node 6 is at a distance $d_{1}$ from node 3 , and $v_{d}$ is closer from this node than $d_{2}$ and $d_{3}$, the candidates of node 3 are nodes 5,6 and $v_{d}$. Likewise it is done for the other nodes.

Figure 8 shows the expected number of transmissions varying the distance $D$ between the source and the destination for a QOO network build as explained before. The curves shown in the Figure have been obtained using a maximum number of candidates per node equal to 1, 2, 3 and 5 (cfr. the numbers in the legend). Figure 9 shows the number of nodes that resulted in the QOO networks used to obtain the corresponding values of Figure 8. In Figure 8 we have also added the lower bounds obtained using Equation (8) (thin lines), and the lower bound for an infinite number of candidates given by Equation (5) (dashed line). 


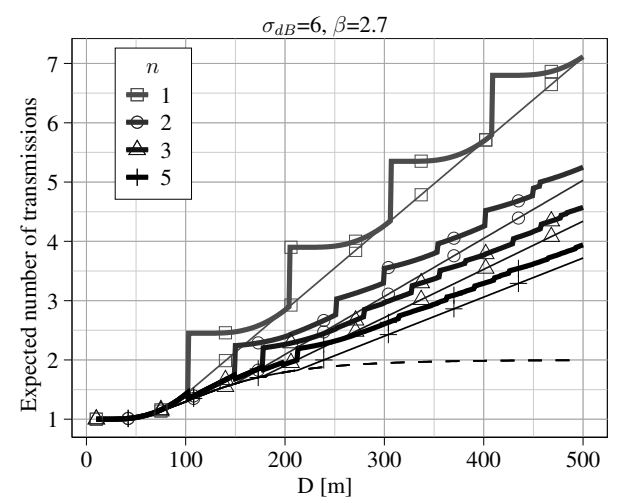

Figure 8: Lower and upper bounds (thin and thick lines respectively) of the minimum expected number of transmissions achievable with OR for $n=1,2$, 3 and 5 maximum number of candidates per node. The dashed line corresponds to infinite number of candidates.

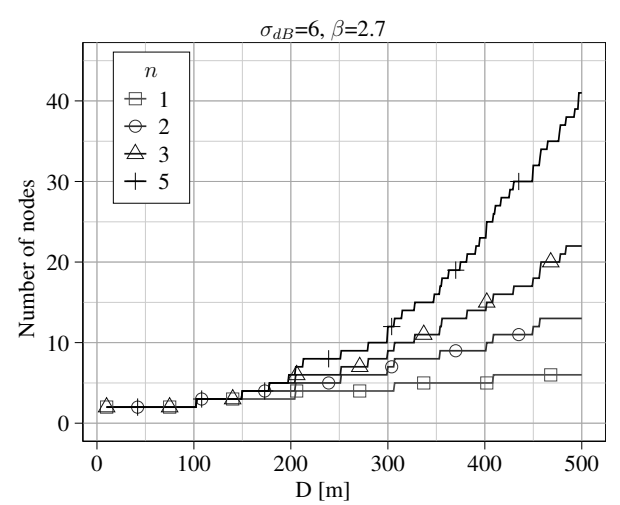

Figure 9: Number of nodes of the quasi optimal OR networks used in Figure 8

The delivery probability of the links $(p(d))$ has been obtained using the shadowing model (Equation (13)) with a path loss exponent $\beta=2.7$. The expected number of transmissions has been obtained using the Markov chain that we have proposed in [10].

Figure 8 confirms that the lower bounds of the expected number of transmissions obtained with Equation (8), especially when $n>1$, are very tight, since they are very close to the upper bound obtained with the QOO network. Furthermore, this result seems to indicate that the MPD are very close to the optimum distances. We shall investigate this in the next section. Note that the discontinuities of the upper bound occur at the distances where a new node is added to the QOO network. For instance in the scenario with 1 candidate, which occurs when the distance between the source and the destination $(D)$ is a multiple of $d_{1}$.

\section{Optimal Distance to Candidates in a Finite Network}

In the previous sections the MPD have been obtained and used to derive bounds, which are rather accurate approximations as well, of the performance of OR measured by the mean number of transmission required to reach the destination.

For a network of finite length $(D<\infty)$, the optimal distances of the candidates -in the sense of minimizing the mean number of transmissions required to reach the destination - are more complex to obtain and in general may not coincide with the MPD

In this section, we use a numerical approximation to find the optimal distances in a finite length network. This is used to confirm some of the intuitions that have been applied previously, and provide a further insight into the optimal distances problem.

Let $V_{n}(x)$ be the minimum mean number of transmissions required to reach the destination that is at distance $x$ from the source node, when a maximum of $n$ candidates per node is used. We can write

$$
\begin{aligned}
V_{n}(x)=\min _{x_{1}<\cdots<x_{n}}\left\{\frac{1}{1-\prod_{i=1}^{n} q\left(x_{i}\right)} \times\right. & \\
& \left.\quad\left(1+p\left(x_{n}\right) V_{n}\left(x-x_{n}\right)+\cdots+\prod_{i=2}^{n} q\left(x_{i}\right) p\left(x_{1}\right) V_{n}\left(x-x_{1}\right)\right)\right\}
\end{aligned}
$$




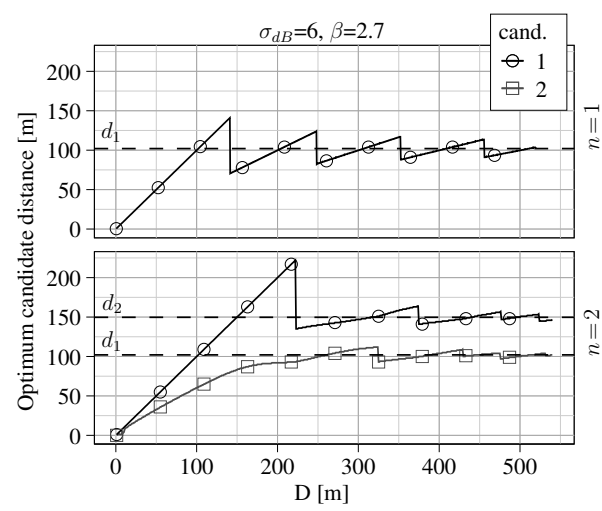

Figure 10: Optimum distances of the candidates $\left(d_{i}^{*}\right)$ with a maximum of $n=1$ and $n=2$ candidates per node (top and bottom respectively). The dashed lines are the MPD $\left(d_{i}\right)$.

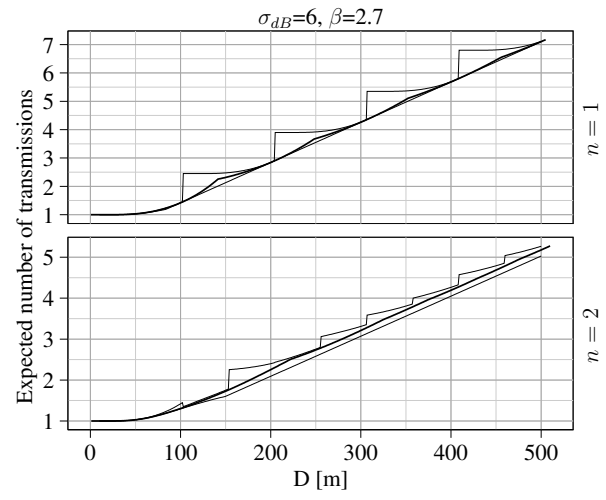

Figure 11: Expected number of transmissions obtained with the optimum distances of Figure 10, and its upper and lower bounds.

where $q(d)=1-p(d)$ and $V_{n}(0)=0$. If the number of nodes between the source and the destination is less than $n$, then the destination and the intermediate nodes are taken as candidates. We shall refer as $d_{i}^{*}$ to the optimal distances $x_{i}$ that minimize Equation (14).

We have solved the optimization problem of (14) in an approximate fashion by considering a discrete network (a finite number of nodes are evenly distributed between source and destination) and then performing an exhaustive optimum search. The network density, i.e., the number of nodes, has been increased until the minimum does not vary significantly. Obviously, the exhaustive search becomes unfeasible as the maximum number of candidates, $n$, or the network size, $D$, grow. For this reason, we have limited this method to a maximum number of candidates equal to $n=1$ and $n=2$. Nevertheless, as we will see in the following, these two scenarios are enough for our purposes.

Figure 10 compares the optimal distances $\left(d_{i}^{*}\right)$ and the MPD $\left(d_{i}\right)$ as functions of $D$. The optimum mean number of transmission obtained for the optimal distances is shown in Fig. 11 along with its corresponding lower and upper bounds.

We observe that the optimal distances converge to the maximum progress distances $\left(d_{1} \approx 102 \mathrm{~m}\right.$ and $\left.d_{2} \approx 150 \mathrm{~m}\right)$ when $D$ grows. It is also observed that while the MPD of the first candidate $\left(d_{1}\right)$ are the same for different values of $D$ and $n$, the optimum distances $d_{1}^{*}$ are different for $n=1$ and for $n=2$, although they converge to the same value (that of the maximum progress distance, $d_{1}$, as we said before).

Notice that, as expected, when $n=1$ and $D$ is a multiple of $d_{1}$, the optimal distance equals the maximum progress distance $\left(d_{1}^{*}=d_{1}\right)$ and the lower bound of $V_{1}(D)$ turns out to yield an exact value. Also, as it has been predicted, the lower bound for $V_{1}(D)$ is tighter than that for $V_{2}(D)$. On the other hand, in both cases $(n=1,2)$ when $D$ grows the shape of $V_{n}(D)$ tends to be a straight line whose slope is matched by that of the lower bound, i.e., by $1 / \mathrm{E}\left[\Delta_{n}^{*}\right]$. Moreover, the shape of $V_{2}(D)$ gets smooth more rapidly than $V_{1}(D)$ does. A similar observation can be made about the rate of convergence of the optimal distances to the MPD.

\section{Node Position in Linear OR Networks}

The MPD computed in Section 2 can be of practical interest in the design of a static network using OR. For example, the back-haul of a mesh network, or the position of the nodes in a sensor network. A first approach could be the Quasi Optimal OR Network 


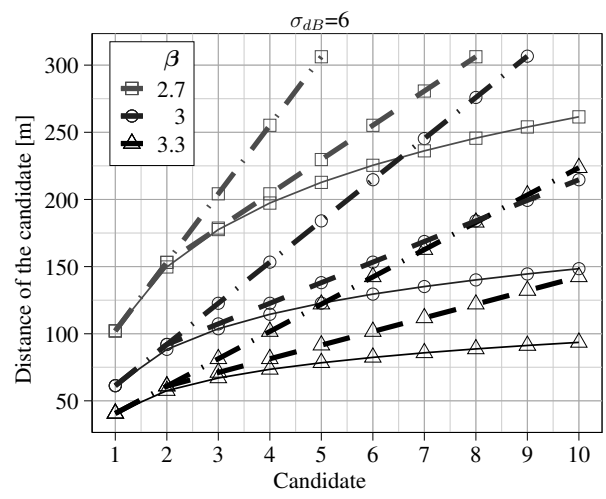

Figure 12: MPD of the candidates (thin lines) and its $d_{1} / 4$ (dashed line) and $d_{1} / 2$ (dot-dashed line) approximations.

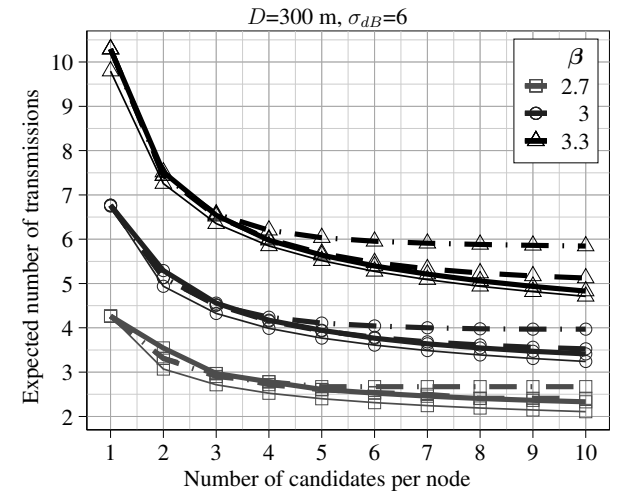

Figure 13: Expected number of transmissions: (i) lower bound (thin lines), (ii) using the QOO network of Section 6 (solid lines), (iii, iv) using, respectively, its $d_{1} / 4$ (dashed line) and $d_{1} / 2$ (dot-dashed line) approximations.

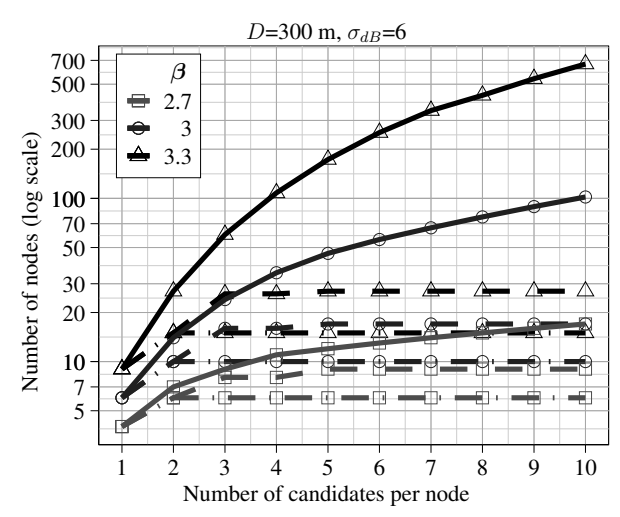

Figure 14: Number of nodes of the networks used in Figure 13 (i) using the QOO network of Section 6 (solid lines), (ii, iii) using its $d_{1} / 4$ (dashed line) and $d_{1} / 2$ (dot-dashed line) approximations.

described in the previous section. However, for such network the number of nodes increases nearly exponentially with the distance between the source and the destination, $D$, as shown in Figure 9. In this section we look for positions of the nodes that, being close to their optimal values, allow reducing the number of nodes of the network.

Looking at the MPD obtained for different parameters of the propagation model (Figure 3 ), we can observe that $d_{2} \approx d_{1}+d_{1} / 2$ and $d_{3} \approx d_{1}+d_{1} / 2+d_{1} / 4$. This suggest that a good compromise is positioning the nodes equally spaced at a distance $d_{1} / 4$, choosing $d_{1}$ for the first candidate, $\hat{d}_{2}=d_{1}+d_{1} / 2$ for the second, and $\hat{d}_{i}=d_{1}+d_{1} / 2+(i-2) \times d_{1} / 4$ for the candidates $i>2$. Doing this way, a distance $D$ would require a number of nodes $N \leq 4 \cdot\left\lceil D / d_{1}\right\rceil$. If only 2 candidates are going to be used, or if we wish to reduce further the number of nodes, a coarser approach would be positioning the nodes equally spaced at a distance $d_{1} / 2$, choosing $d_{1}$ for the first candidate and $\hat{d}_{i}=d_{1}+(i-1) \times d_{1} / 2$ for the candidates $i>2$. Doing this way, the required number of nodes would be $N \leq 2 \cdot\left\lceil D / d_{1}\right\rceil$. We shall refer to these approximations as $d_{1} / 4$ and $d_{1} / 2$ respectively. Figure 12 shows the MPD computed as in Section 2 and its $d_{1} / 4$ and $d_{1} / 2$ approximations.

Figures 13 and 14 show the sensitivity of the expected number of transmissions to the $d_{1} / 4$ and $d_{1} / 2$ approximations. Here we have used the same settings as in Section 5: $D=300 m ; \beta=2.7,3$ and 3.3. For each value of $\beta$ Figure 13 shows four curves of the expected number of transmissions: (i) the lower bound computed as in Section 3 (note 
that these curves are the same than those shown in Figure 5); (ii) using the QOO network of Section 6 (solid lines); and (iii, iv) using its $d_{1} / 4$ (dashed line) and $d_{1} / 2$ (dot-dashed line) approximations. Figure 14 shows the number of nodes of the networks that where used to compute the expected number of transmissions for the corresponding cases (ii, iii, iv) of Figure 13.

As shown in Figure 13 the expected number of transmissions obtained for the QOO network is very close to the lower bound. Nevertheless, Figure 14 shows that building the QOO network requires a high number of nodes. The maximum value is 665 nodes, obtained for $\beta=3.3$ (where the nodes' coverage is the shortest) and 10 candidates per node. Figure 13 also shows that the expected number of transmissions obtained for the $d_{1} / 4$ and $d_{1} / 2$ approximations it is very close to the lower bound too. Only for $\beta=3.3$ and more than 5 candidates per node the difference is noticeable. However, in Figure 14 we can see that the number of nodes using the $d_{1} / 4$ and $d_{1} / 2$ approximations is enormously reduced (e.g. it is 27 and 15 nodes respectively for the $d_{1} / 4$ and $d_{1} / 2$ approximations in the same scenario for which 665 nodes are used with the QOO network).

We conclude that choosing the position of the 2 candidates closest to the sender near to their optimal positions, is the most critical in order to minimize the expected number of transmissions. Consequently, what we have called $d_{1} / 2$ approximation may be a sensible rule of thumb in the design of the node positions in a linear static network using OR routing. In summary, the $d_{1} / 2$ approximation yields a good trade-off between performance and number of nodes when we want to deploy a linear network.

\section{Node Positioning in Two-dimensional Multihop Networks}

In this section we investigate whether the $d_{1} / 2$ approximation proposed in previous sections can also be used in a two-dimensional network. We consider a square grid with $N=k \times k$ nodes, and denote by $d$ the distance between two adjacent nodes on the square side (see Figure 15). Clearly, the best positions for those candidates to reach destinations located in verticals or horizontals lines departing from the source will be the MPD. Thus, setting $d=d_{1} / 2$ would be a good rule of thumb for these destinations. However, most destinations are not located in these lines. Thus, it is not clear whether another value for $d$ may be a better choice.

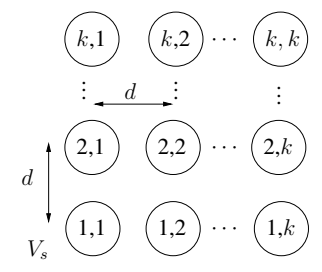

Figure 15: Grid topology.

In order to investigate an appropriate value for $d$ we have varied its value, while maintaining the number of nodes of the grid, $N$. We have assumed that the source $V_{s}$ and the destination $V_{d}$ are located at positions $(1,1)$ and $(i, k)$ where $i \in\{1, \cdots, k\}$, respectively (see Figure 15). Furthermore, we refer to $D$ as the distance between $V_{s}$ and $V_{d}$. We have used a semi-optimum candidate selection algorithm, DPOR [17], to select the candidates of the nodes towards the destination. DPOR is a fast and efficient candidate selection algorithm whose performance is very close to the optimum algorithms proposed in the literature, whereas it runs much faster (see [17]). The link delivery probabilities 


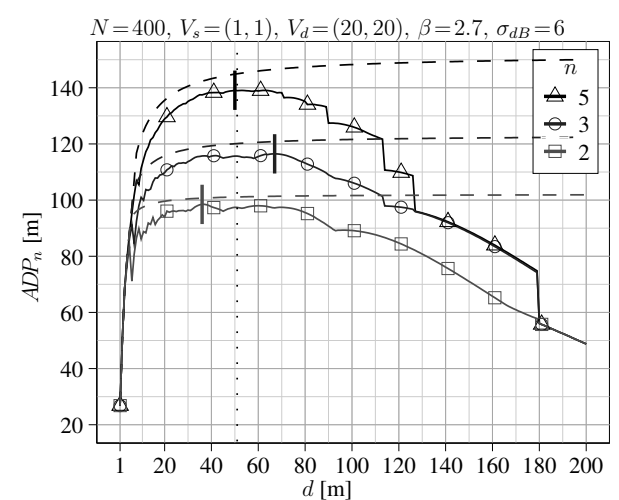

Figure 16: $A D P$ using DPOR for $V_{s}=(1,1)$ and $V_{d}=(20,20)$, varying the distance between two adjacent nodes on the square side.

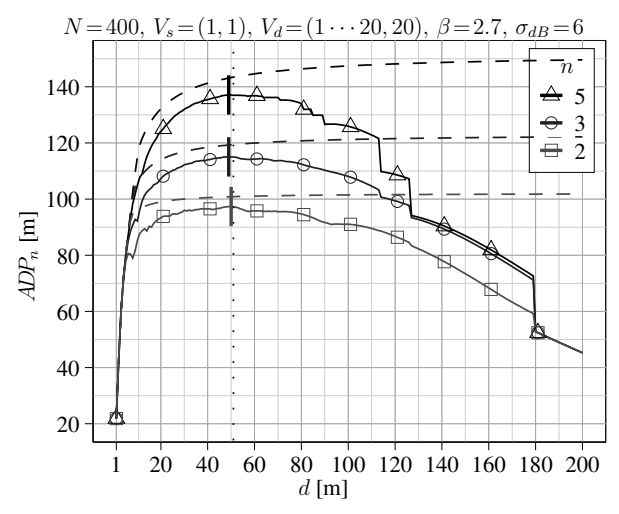

Figure 17: Mean value of $A D P$ using DPOR for $V_{s}=$ $(1,1)$ and $V_{d}=(1 \cdots 20,20)$, varying the distance between nodes on the square side.

between two nodes have been assigned with the shadowing model with $\beta=2.7$ and $\sigma_{d b}=6.0$. We have assumed that a link between any two nodes exists only if the delivery probability between them is greater (or equal) than min.dp $=0.1$.

Note that varying $d$, the value of $D$ and possibly the nodes chosen by the candidate selection algorithm also change. Thus, in order to compare the goodness of the different values of $d$, we have defined the metric that we call Average Distance Progress (ADP) as:

$$
A D P_{n}\left(d, V_{s}, V_{d}\right)=\frac{D\left(d, V_{s}, V_{d}\right)}{\operatorname{EAX}_{n}\left(d, V_{s}, V_{d}\right)}
$$

where $n$ is maximum number of candidates per node, $D\left(d, V_{s}, V_{d}\right)$ is the distance from the source $V_{s}$ to the destination $V_{d}$, and $E A X_{n}\left(d, V_{s}, V_{d}\right)$ is its $E A X_{n}$ using the candidates chosen for a given value of $d$. Note that $A D P_{n}\left(d, V_{s}, V_{d}\right)$ represents the average number of meters that a packet progress towards $V_{d}$ at each transmission shot. Therefore, the optimum value of $d$ would maximize Equation (15).

Figure 16 shows the value of $A D P_{n}\left(d, V_{s}, V_{d}\right)$ for $N=400$ nodes varying the distance between adjacent nodes on the square side in the range $1 \leq d \leq 200 \mathrm{~m}$. The value of $E A X_{n}$ has been computed using the EAX recursive formula. The curves have been obtained for different maximum number of candidates: $n=\{2,3,5\}$. In Figure 16 we have fixed the position of the source and the destination at the diagonal end points, i.e. $V_{s}=(1,1)$ and $V_{d}=(20,20)$. For the sake of comparison, we have included the value of $d_{1} / 2$ (see vertical dotted line in Figure 16). For a shadowing propagation model with the parameters used in Figure 16 it is $d_{1} / 2 \approx 51 \mathrm{~m}$ (see Figure 3 ).

For each $n$, the thick and solid vertical lines in Figure 16 show the values of $d$ that maximize $A D P_{n}$. Figure 16 also shows an upper bound of $A D P_{n}$ (dashed line) obtained using the value of $E A X_{n}$ computed by means of Equation (8). Note that in the grid topology the nodes are not located at their optimal positions. Therefore, the fact that the maximum value obtained for $A D P_{n}$ is close to the upper bound shows that the performance that can be obtained with OR is not very sensitive to deviations in the candidates placement with respect to their optimal positions. This fact was already observed in Section 8 for a linear setting.

Figure 16 shows that the larger the maximum number of candidates $(n)$, the higher the value of ADP is. Additionally, Figure 16 shows that the curves of ADP is rather flat around their maximum point. For instance, with $n=2, A D P_{2}$ reaches its maximum 
$(101 \mathrm{~m})$ at $d=36 \mathrm{~m}$. However, using $d=d_{1} / 2 \approx 51 \mathrm{~m}$ it is obtained that $A D P_{2}=97 \mathrm{~m}$, which is only $5 \%$ smaller than the maximum.

Figure 16 shows that the optimum $d$ is smaller than $d=d_{1} / 2 \approx 51 \mathrm{~m}$ for $n=2$, while it is higher for $n=\{3,5\}$. The reason is the following. Recall that the optimum position of the first candidate is $d_{1}$, and the second is very close to $d_{1} / 2$. Therefore, choosing $d=\left(d_{1} / 2\right) / \sqrt{2} \approx 36 \mathrm{~m}$ we obtain the best position for the candidates in the diagonal. If more than 2 candidates are used, the best position of the other candidates is not well fitted by nodes in the diagonal. Thus, the candidate selection algorithm also chooses nodes out of the diagonal, and closer to the sending node than their MPDs (since they are better than nodes located farther). Being the candidates closer to $V_{s}$ than their optimal positions motivates that a slightly higher performance can be achieved using a higher value for $d$, as shown in Figure 16 .

Figure 17 shows the average value of ADP over all destinations in one side of the square opposite to the source, i.e. $V_{d}=(i, 20), i \in\{1,2, \cdots, 20\}$. As before, the vertical lines are located at $d=d_{1} / 2 \approx 51 \mathrm{~m}$ (dotted) and the values of $d$ that maximize ADP, and the horizontal dashed lines are the upper bounds of $A D P_{n}$, obtained using the value of $E A X_{n}$ computed by means of Equation (8). Interestingly, Figure 17 shows that taking the average all vertical lines almost overlap, coinciding with $d=d_{1} / 2$.

We can conclude that in a grid topology, ADP is practically insensitive to $d$ over a wide interval. Additionally, setting $d=d_{1} / 2$ is a good rule of thumb, yielding an ADP close to the optimum for all possible destinations.

To further study the $d_{1} / 2$ approximation we have run another experiment with a grid of $N=10 \times 10$ nodes. We have used the same conditions as before, thus $d=d_{1} / 2 \approx 51 \mathrm{~m}$. Like in the previous scenario, we have fixed the position of the source to $V_{s}=(1,1)$. The position of the destination is varied by choosing the nodes on the diagonal, i.e. $V_{d}=(i, i), 2 \leq i \leq 10$. Due to the reduced size of the network, for the candidate selection now we have run an optimum algorithm (MTS [18]). The maximum number of candidates has been set equal to $n=5$.

Figure 18 shows the candidates sets that are chosen for each destination. This is done by showing the distance from the source node $V_{s}$ to each of the 5 selected candidates vs. the distance between source and the destination. It turns out that all candidates are chosen among the nodes located in the three central diagonals of the grid (i.e, $(i, j)|i-j| \leq 1$ ). In order to identify these nodes, their distance to $V_{s}$ is indicated with the labels $(i, j)$ on the right side of the plot (note that the distance of nodes $(i, j)$ and $(j, i)$ to $V_{s}$ is the same). For the sake of comparison, the figure also shows the MPD (dashed lines), with the labels $d_{i}$ on the right. For each destination in Figure 18, which shows the $A D P_{5}$ (solid line), and its upper bound (dashed line), obtained as in Figures 16 and 17.

We can see in Figure 18 that for different positions of the destination close to the source $\left(V_{d}=(i, i), 2 \leq i \leq 7\right)$, different candidates sets are chosen in the neighborhood of the source. For further distances $\left(V_{d}=(i, i), i>7\right)$, the candidates set remains the same. The geographical positions of the candidates in this set are depicted in Figure 20. Figure 20 also shows the MPDs (dashed lines), with labels $d_{i}$. Note that candidates located at the MPDs would be in the intersection of these dashed lines with the segment connecting the source and the destination (dotted line in Figure 20 for the destinations located in the diagonal of the grid). Figure 20 shows that the candidates chosen by the optimal algorithm, MTS, are indeed those closest to the MPDs. Figure 20 also gives a pictorial view about how far are the chosen candidates from their optimal position. Note that choosing the destination in the diagonal is a worst case, since the grid is built 


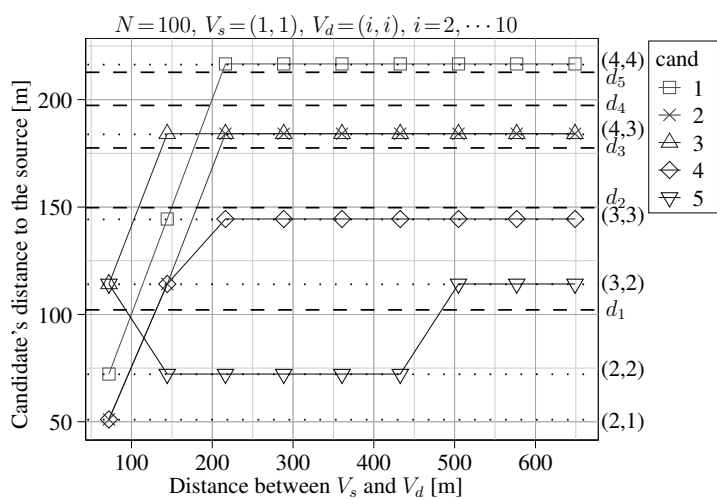

Figure 18: Geographic distance of the optimum candidates in the grid to the source for $V_{s}=(1,1)$, varying the destination to $V_{d}=(i, i), 2 \leq i \leq 10$.

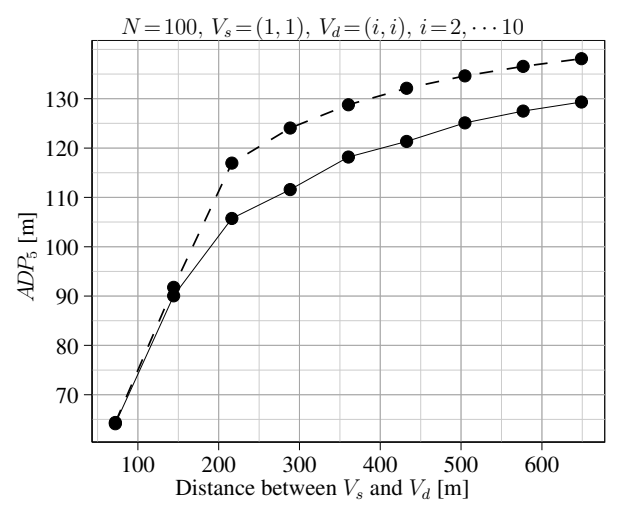

Figure 19: ADP and upper bound (dashed line) obtained for each candidate set depicted in figure 18 .

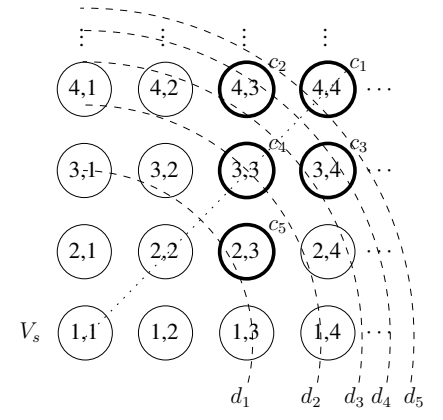

Figure 20: Candidates of figure 18 chosen for $V_{d}=(i, i), i>7$ (circles with thick lines). Dashed lines with labels $d_{i}$ show the maximum progress distances from the source, $V_{s}$.

applying the $d_{1} / 2$ rule of thumb for the nodes located in verticals or horizontals lines departing from the source.

Recall that the upper bound shown in Figure 19 is obtained using equation (8). For the first two points $\left(V_{d}=\{(2,2),(3,3)\}\right)$, the destination is close to the source, and the first component of equation $(8)$ applies $(E A X \approx 2-p(D))$. For the other points $\left(V_{d}=(i, i), i>3\right)$, the second component of equation (8), is used. Recall that in this case equation (8) assumes the candidates located at the MPD. The MPD are computed for a destination far from the source. In fact, we observe in Figure 19 that for the points corresponding to $V_{d}=(i, i), i>3$ the difference between the bound and the measured $\mathrm{ADP}$ is reduced slightly when the distance between the source and destination increases. However, the fact that this difference is small shows that the MPDs are also a good estimation when the destination is relatively close to the source. Additionally, Figure 19 shows that for $V_{d}=(10,10)$ it is obtained $A D P \approx 129$ with an upper bound $\approx 138$, thus, about only $6.7 \%$ relative error. As explained above, choosing the destination in the diagonal is a worst case. Therefore, this small error shows again that the performance is not very sensitive to deviations from the optimal position of the candidates. Summing up, we conclude that choosing $d=d_{1} / 2$ is a good rule of thumb for building the grid.

\section{Related Work}

The majority of previous studies that evaluate the performance of opportunistic routing do not use analytical methods, instead they resort to simulations or empirical measurements $[5,19,20,21,22,23]$. On the other hand, most of the works are devoted to 
the selection of the candidates, the way of acknowledging packet reception and how to prevent, or at least reduce, duplicate transmissions.

Biswas and Morris $[4,5]$ designed and implemented Extremely Opportunistic Routing (ExOR) for wireless multihop networks. It selects the candidates set according to route costs based on Expected Transmission Count (ETX) [24]. In [8, 25] Zhong et al. proposed the expected anypath transmission (EAX) as a new metric for OR that generalizes the ETX, and proposed a candidate selection and prioritization algorithm based on it. In addition to ETX and EAX, some OR schemes such as geographic opportunistic routing (GOR) [26] and Contention-Based Forwarding (CBF) [27] considers geographic distance as metric. Under some scenarios, relay nodes are assumed to be aware of their positions. Therefore, routing can be done by selecting the candidate, which is geographically closest to the destination.

Dubois-Ferrière et al. [28] introduced a shortest anypath algorithm that finds optimal candidates sets. The authors generalized the well-known Bellman-Ford algorithm for anypath routing and proved its optimality, but the resulting algorithm has exponential computational running time. They provided another optimal polynomial-time algorithm in [29] to jointly select the optimal candidates set. They also extended their model proposed in $[28,9]$ to a multi-rate scenario. In [18] the key problem of how to optimally select the candidates sets is addressed, and an optimal algorithm that minimizes the expected total number of transmissions is developed. The authors in [30] provided an analytical framework to model the problem of selecting the optimal candidates set for both the constrained and unconstrained candidates set selection. They proposed two algorithms for optimal candidates set selection, one for the constrained and one for the unconstrained case.

In the recent work from [31], the authors presented a bandwidth-aware opportunistic routing (BOR) with admission control protocol. They proposed a new metric to determine the priority of candidates in the candidates set. The new metric analyzes the expected available bandwidth (EAB) and the expected transmission cost (ETC) using OR. The candidate with a high bandwidth and low transmission cost will relay the packet with a higher priority. They devised an admission control mechanism to reject traffic flows with a high bandwidth requirement that cannot be satisfied by the discovered opportunistic path. This mechanism guarantees that the QoS of ongoing traffic is maintained. Authors in [32] analyzed the opportunistic routing gain under the presence of link correlation considering the loss of data and acknowledgment packets. They defined a new metric that captures the expected number of anypath transmissions under the effect of link correlation. Based on the new metric they proposed a candidate selection algorithm. Their results show that considering the correlation between the links improved the expected number of transmissions from the source to the destination.

Using multiple next-hop candidates however faces the challenge of avoiding duplicate forwarding, which occurs when more than a candidate relays the same packet. This issue is usually called as candidate coordination which is one of the important issues of OR. There are different algorithms with different performances for the candidate coordination. In [7] authors surveyed opportunistic routing issues with emphasize on the candidate coordination algorithms.

ExOR [4] uses a modified version of the 802.11 MAC for candidate coordination. It reserves multiple slots of time for the receiving candidates to return ACKs. Each ACK indicates that the packet is successfully received by the candidate. In addition, it contains the IDs of the successful recipients with the higher priority known to the ACKs sender. 
All candidates listen to all ACK slots before deciding whether to forward; this is done in case a low-priority candidates ACK reports a higher-priority candidate's ID whose ACK was not correctly received.

Authors in [33] showed how in the ACK-based method if the highest priority candidate fails to receive the data packet, the channel will then be idle for a period of time longer than the DIFS. It may happen that some other nodes, from another flow, not hearing the data packet, would send their packets; this would cause a collision with subsequent ACKs, if any, from the lower priority candidates. The authors proposed FSA [33] to provide the coordination between the candidates with lower delay than ACK-based method. FSA is only based on a single ACK which is sent through the highest priority candidate that has received the packet successfully.

Combining the idea of OR with Network Coding (NC) [34] can provide an elegant method for preventing duplicate transmissions without coordinating the candidates by coding the packets $[35,36,37]$. MAC-independent Opportunistic Routing \& Encoding (MORE) [35] was proposed by Chachulski et al. The data flow is divided in some batches which contain a certain amount of packets. MORE adds the candidates set to the packet's header and broadcasts the coded packet. The receiving node checks whether its ID is in the candidates set. Furthermore, the node checks whether the packet is linearly independent of the packets it has received before. If so, it creates a new coded packet by linearly combining the received packets and rebroadcasting it. The simulations results show that MORE can significantly improve the network throughput in comparison to ExOR; and, it can also eliminate the requirement of global coordination among candidates. A practical $\mathrm{NC}$ mechanism that enables the supporting of efficient unicast communication in wireless mesh networks is COPE [38]. By using OR, COPE enables each node to learn about local state information. The CORE [39] is a coding-aware OR mechanism that combines OR and localized inter-flow $\mathrm{NC}$ for improvement of the throughput performance in a wireless mesh network. Through OR, CORE allows the next-hop node with the most coding gain to continue packet forwarding. Through localized NC, CORE attempts to maximize the number of packets that can be carried in a single transmission

Authors in [40] developed an anycast mechanism at the link layer for wireless ad hoc networks. They used explicit control packet(s) exchanged immediately before sending a data packet. In this approach the sender multicasts the Request-To-Send (RTS) to the its candidates set. The RTS contains all the candidates addresses which are ordered according to a metric. When an intended candidate receives the RTS packet, it responds by a Clear-To-Send (CTS). These CTS transmissions are sent in decreasing order of candidate priority.the first When the sender receives a CTS, it transmits the DATA packet to the sender of this CTS after a Short Inter-frame Space (SIFS) interval. All such receivers then set their Network Allocation Vector (NAV) until the end of ACK period. This mechanism is guaranteed to have a single winner and it can avoid duplicate transmissions. In [41] a method for avoiding duplicate forwarding in opportunistic routing is proposed which does not depend on any form of information exchange between next-hop candidates. The proposed technique enables forwarding nodes to control relaying at their neighbors on a per-packet basis using a small amount of information piggybacked on packets. The authors compared their proposal with ExOR and demonstrated that their proposal improves throughput of the network by reducing unnecessary transmissions.

There are some papers which propose analytical models to study the performance of OR. In [42] Shah et al. presented a framework to model OR for low loaded sensor networks. They also explored the performance of opportunistic routing for different node densities, 
channel quality and traffic rates, and compared it with geographic routing. They also identified optimal points for the duty cycle of nodes that minimize the power consumption. Baccelli et al. [43] aimed at quantifying and optimizing the potential performance gains of opportunistic routing strategies compared with classical routing schemes. Their analysis was under the assumptions of Aloha-based MAC layer. Zubow et al. in [22] claimed that shadow fading losses for spatially close candidates are not independent from each other, unlike commonly assumed. They presented measurements obtained from an indoor testbed and concluded that correlations can not be neglected if nodes are separated by less than $2 \mathrm{~m}$. In [44] an analytical approach for studying OR in wireless multi-hop networks have been proposed. They used lognormal shadowing and Rayleigh fading models for packet reception. In their model they assume that the nodes are uniformly distributed over the plane. The authors did not consider any specific candidate selection algorithm, but simply compute the expected progress of the packet transmissions based on the probability of any node in the progressing region successfully receives the packet. They extended their work by using directional antennas and different radio propagation models and spatial node distributions in [45]. In [46] an algebraic approach was applied to study the interaction of OR routing algorithms and routing metrics. They showed that OR in combination with ETX could degrade the performance of network. Authors in [26] analyzed the trade-off among the packet advancement, reliability and MAC coordination time cost in geographic opportunistic routing. They proposed a local metric, expected one-hop throughput (EOT), to balance the trade-off between the packet advancement and the cost of candidate coordination. They showed that although having more candidates brings more chances for the packet to get closer to the destination and be delivered, the gained benefit is marginal.

\section{Conclusions}

In this paper we have derived the equations that yield the distances of the candidates in Opportunistic Routing (OR) such that the per transmission progress towards the destination is maximized. We have called them as the maximum progress distances (MPD). The only ingredient to obtain these distances is the law for the delivery probability between nodes as a function of distance. An important consequence of our derivation is that the the MPD for the already existing candidates do not change if we decide to add a new candidate to the candidate set. We have also observed that, while the MPD vary when the propagation conditions change, the delivery probabilities to the candidates located at the MPD remain approximately constant. Thus, to select the candidates, or to place the nodes of the back-haul of a mesh network during the network design, we may be implement a method based on the measurement of the delivery probabilities.

Based on these MPD, we have proposed a lower bound to the expected number of transmissions needed to send a packet using OR. The lower bound has proven to be very tight.

By modeling the delivery probabilities with a shadowing propagation model we obtained numerical results showing that the expected number of transmissions can be reduced up to a $30 \%$ with only 2 candidates, whereas in order to reduce it another $30 \%$ the number of candidates has to be increased up to 10 .

We have constructed a quasi optimum OR network locating the nodes and their candidates at the MPD whenever possible. This quasi optimum OR network is used to obtain an upper bound for the expected number of transmissions. This upper bound happens to be very close to our lower bound, which confirms that it is indeed very tight. We have 
further validated these results by building a dense network and computing the optimal distances of the candidates by an exhaustive optimum search. We have seen that the optimal distances of the candidates converge rapidly to the MPD as the length of the network increases.

We have also investigated the sensitivity of the performance to the position of the candidates. Our results allowed us to conclude that choosing the distance of the first two candidates near to their optimal positions is the most critical aspect in order to minimize the expected number of transmissions. Based on this result, we have used the MPD to provide a rule of thumb for placing the nodes in a static network using OR. Compared to the optimal layout, this method will slightly increase the average number of transmissions while the total number of nodes required is reduced enormously. This can be of practical interest in the design of the back-haul of a mesh network, or in the positioning of the nodes in a sensor network.

Finally, we have investigated the maximum performance of OR in two-dimensional scenarios. Our results revealed that a network design with grid layout yields a performance that, for all practical purposes, is very close the optimum. Furthermore, in the grid design, adjusting the shortest distance between two adjacent nodes to its optimal value is not a critical aspect. In our experiments the sensibility of the performance with respect to this design parameter has proven to be rather low in a wide region around its optimal value.

\section{Acknowledgments}

This work was supported by the Spanish Ministerio de Ciencia e Innovación through the projects TIN2010-21378-C02-01 and TIN2010-21378-C02-02 and by the Generalitat de Catalunya through project 2009-SGR-1167.

[1] I.F. Akyildiz and Xudong Wang. A survey on wireless mesh networks. Communications Magazine, IEEE, 43(9):S23 - S30, September 2005.

[2] Ian F. Akyildiz and Xudong Wang. Wireless Mesh Networks. John Wiley \& Sons Ltd, 2009.

[3] Ming Li Kai Zeng, Wenjing Lou. Multihop Wireless Networks: Opportunistic Routing. John Wiley \& Sons, 2011.

[4] Sanjit Biswas and Robert Morris. Opportunistic routing in multi-hop wireless networks. ACM SIGCOMM Computer Communication Review, 34(1):69-74, 2004.

[5] S. Biswas and R. Morris. ExOR: opportunistic multi-hop routing for wireless networks. ACM SIGCOMM Computer Communication Review, 35(4):133-144, 2005.

[6] Haitao Liu, Baoxian Zhang, H. Mouftah, Xiaojun Shen, and Jian Ma. Opportunistic routing for wireless ad hoc and sensor networks: Present and future directions. Communications Magazine, IEEE, 47(12):103-109, December 2009.

[7] Che-Jung Hsu, Huey-Ing Liu, and Winston Khoon Guan Seah. Opportunistic routing - a review and the challenges ahead. Computer Networks, 55(15):3592-3603, 2011.

[8] Zifei Zhong, Junling Wang, Srihari Nelakuditi, and Guor-Huar Lu. On selection of candidates for opportunistic anypath forwarding. SIGMOBILE Mob. Comput. Commun. Rev., 10(4):1-2, 2006. 
[9] H. Dubois-Ferriére, M. Grossglauser, and M. Vetterli. Valuable detours: Least-cost anypath routing. Networking, IEEE/ACM Transactions on, 19(2):333-346, April 2011.

[10] Amir Darehshoorzadeh, Llorenç Cerdà-Alabern, and Vicent Pla. Modeling and comparison of candidate selection algorithms in opportunistic routing. Computer Networks, 55(13):2886-2898, 2011.

[11] Hideaki Takagi and Leonard Kleinrock. Optimal transmission ranges for randomly distributed packet radio terminals. Communications, IEEE Transactions on, $32(3): 246-257,1984$.

[12] Ivan Stojmenovic. Position-based routing in ad hoc networks. IEEE Communications Magazine, 40(7):128-134, 2002.

[13] Brad Karp and Hsiang-Tsung Kung. Gpsr: Greedy perimeter stateless routing for wireless networks. In Proceedings of the 6th annual international conference on Mobile computing and networking, pages 243-254. ACM, 2000.

[14] Michele Zorzi and Ramesh R Rao. Geographic random forwarding (geraf) for ad hoc and sensor networks: multihop performance. IEEE Transactions on Mobile Computing, 2(4):337-348, 2003.

[15] P. Cardieri. Modeling interference in wireless ad hoc networks. Communications Surveys \& Tutorials, IEEE, 12(4):551-572, 2010.

[16] The Network Simulator ns-2.

[17] Amir Darehshoorzadeh and Llorenç Cerdà-Alabern. Distance progress based opportunistic routing for wireless mesh networks. In Wireless Communications and Mobile Computing Conference (IWCMC), 2012 8th International, pages 179-184, August 2012.

[18] Yanhua Li, Wei Chen, and Zhi-Li Zhang. Optimal forwarder list selection in opportunistic routing. In Mobile Adhoc and Sensor Systems. MASS '09. IEEE 6th International Conference on, pages 670-675, October 2009.

[19] Y. Yuan, H. Yang, S.H.Y. Wong, S. Lu, and W. Arbaugh. ROMER: Resilient opportunistic mesh routing for wireless mesh networks. In IEEE Workshop on Wireless Mesh Networks (WiMesh), 2005.

[20] E. Rozner, J. Seshadri, Y. Mehta, and L. Qiu. Simple opportunistic routing protocol for wireless mesh networks. In 2nd IEEE Workshop on Wireless Mesh Networks, WiMesh, pages 48-54, 2006.

[21] A. Zubow, M. Kurth, and JP Redlich. Multi-channel opportunistic routing. In European Wireless Conference (EW), 2007.

[22] M. Kurth, A. Zubow, and J.-P. Redlich. Cooperative opportunistic routing using transmit diversity in wireless mesh networks. In IEEE INFOCOM, pages 1310-1318, 2008 . 
[23] J. Wu, M. Lu, and F. Li. Utility-based opportunistic routing in multi-hop wireless networks. In Distributed Computing Systems. ICDCS'08. The 28th International Conference on, pages 470-477, 2008.

[24] Douglas S. J. De Couto, Daniel Aguayo, John Bicket, and Robert Morris. A highthroughput path metric for multi-hop wireless routing. Wireless Networks, 11(4):419434, 2005.

[25] Zifei Zhong and S. Nelakuditi. On the efficacy of opportunistic routing. In SECON '07, pages 441-450, June 2007.

[26] Kai Zeng, Wenjing Lou, Jie Yang, and Donald R. Brown, III. On throughput efficiency of geographic opportunistic routing in multihop wireless networks. Mob. Netw. Appl., 12:347-357, December 2007.

[27] Holger Füßler, Jörg Widmer, Michael Käsemann, Martin Mauve, and Hannes Hartenstein. Contention-based forwarding for mobile ad hoc networks. Ad Hoc Networks, 1(4):351-369, 2003.

[28] H. Dubois-Ferriere, M. Grossglauser, and M. Vetterli. Least-cost opportunistic routing. In Proceedings of 2007 Allerton Conference on Communication, Control, and Computing, 2007.

[29] R. Laufer, H. Dubois-Ferriere, and L. Kleinrock. Polynomial-time algorithms for multirate anypath routing in wireless multihop networks. Networking, IEEE/ACM Transactions on, 20(3):742-755, June 2012.

[30] A.S. Cacciapuoti, M. Caleffi, and L. Paura. Optimal constrained candidate selection for opportunistic routing. In Global Telecommunications Conference (GLOBECOM 2010), 2010 IEEE, pages 1-5, December 2010.

[31] Peng Zhao, Xinyu Yang, Jiayin Wang, Benyuan Liu, and Jie Wang. BOR/AC: Bandwidth-aware opportunistic routing with admission control in wireless mesh networks. In INFOCOM, 2012 Proceedings IEEE, pages 2701-2705, March 2012.

[32] A. Basalamah, Song Min Kim, Shuo Guo, Tian He, and Y. Tobe. Link correlation aware opportunistic routing. In INFOCOM, 2012 Proceedings IEEE, pages 30363040, March 2012.

[33] Z. Yang, K. Zeng, and W. Lou. FSA: A Fast Coordination Scheme for Opportunistic Routing. In 2009 IEEE International Conference on Communications, pages 1-5. IEEE, June 2009.

[34] R. Ahlswede, Ning Cai, S.-Y.R. Li, and R.W. Yeung. Network information flow. Information Theory, IEEE Transactions on, 46(4):1204-1216, July 2000.

[35] Szymon Chachulski, Michael Jennings, Sachin Katti, and Dina Katabi. Trading structure for randomness in wireless opportunistic routing. In SIGCOMM, pages 169-180, New York, NY, USA, 2007. ACM.

[36] R. Bruno and M. Nurchis. Survey on Diversity Based Routing in Wireless Mesh Networks: Challenges and Solutions. Computer Communications, 33(3):269-282, 2010. 
[37] Yan Yan, Baoxian Zhang, H.T. Mouftah, and Jian Ma. Practical coding-aware mechanism for opportunistic routing in wireless mesh networks. In Communications, 2008. ICC '08. IEEE International Conference on, pages 2871-2876, May 2008.

[38] Sachin Katti, Hariharan Rahul, Wenjun Hu, Dina Katabi, Muriel Médard, and Jon Crowcroft. XORs in the air: practical wireless network coding. IEEE/ACM Trans. Netw., 16(3):497-510, 2008.

[39] Y. Yan, B. Zhang, J. Zheng, and J. Ma. Core: a coding-aware opportunistic routing mechanism for wireless mesh networks [accepted from open call]. Wireless Communications, IEEE, 17(3):96-103, June 2010.

[40] Shweta Jain and Samir R. Das. Exploiting path diversity in the link layer in wireless ad hoc networks. Ad Hoc Networks, 6(5):805-825, 2008.

[41] Jihoon Myung and Wonjun Lee. Eliminating duplicate forwarding in wireless opportunistic routing. Communications Letters, IEEE, 16(4):510-513, April 2012.

[42] R.C. Shah, S. Wietholter, and A. Wolisz. Modeling and analysis of opportunistic routing in low traffic scenarios. In WiOpt, pages 294-304, 2005.

[43] F. Baccelli, B. Blaszczyszyn, and P. Muhlethaler. On the performance of time-space opportunistic routing in multihop mobile ad hoc networks. In WiOpt, pages 307-316, April 2008.

[44] Chun-Pong Luk, Wing-Cheong Lau, and On-Ching Yue. An analysis of opportunistic routing in wireless mesh network. In Communications, 2008. ICC '08. IEEE International Conference on, pages 2877-2883, May 2008.

[45] Wing-Cheong Lau Chun-Pong Luk and On-Ching Yue. Opportunistic routing with directional antennas in wireless mesh networks. In IEEE INFOCOM, 2009.

[46] Mingming Lu and Jie Wu. Opportunistic routing algebra and its applications. In IEEE INFOCOM, pages 2374-2382, 2009. 